\title{
AVALIAÇÃO DAS HABILIDADES PRAGMÁTICAS E SOCIAIS EM CRIANÇAS COM DISTÚRBIO ESPECÍFICO DE LINGUAGEM
}

\author{
Evaluation of social and pragmatic skills in children \\ with specific language disorder
}

\author{
Fabiana Cristina Carlino (1), Maria da Piedade Resende da Costa ${ }^{(2)}$, \\ Dagma Venturini Marques Abramides ${ }^{(3)}$
}

\begin{abstract}
RESUMO
Objetivo: avaliar as habilidades sociais de comunicação e pragmáticas em crianças com Distúrbio Específico de Linguagem. Método: participaram do estudo 18 crianças, entre sete e nove anos, sendo que metade $(n=9)$ constituiu o grupo experimental $(G E)$ e a outra metade $(n=9)$ constituiu o grupo controle GC. Ambos os grupos foram formados por três meninas e seis meninos. As crianças foram avaliadas quanto às habilidades pragmáticas por meio de uma filmagem de fala espontânea. Em seguida, filmadas em situação estruturada de interação com um adulto desconhecido do sexo feminino. As respostas foram classificadas em categorias e agrupadas em Repostas Adequadas (RA) e Repostas Inadequadas (RI), sempre de acordo com o contexto comunicativo estabelecido. Resultados: foi possível observar que quanto maior a limitação em habilidades pragmáticas, pior a iniciativa e desempenho nas relações interpessoais. Houve uma diferença significante entre os grupos GE e GC, mostrando que o GE apresentou desempenho inferior ao GC tanto em habilidades pragmáticas quanto sociais. Conclusão: ressalta-se a importância de elaborar métodos de intervenção que busquem tanto a melhoria nos aspectos de fala e linguagem quanto nas relações interpessoais.
\end{abstract}

DESCRITORES: Comunicação; Desenvolvimento da Linguagem; Linguagem Infantil; Relações Interpessoais

\section{INTRODUÇÃO}

Ao se desenvolver, a criança adquire e utiliza funções comunicativas mais interativas, que controlam ou dirigem o comportamento do outro, sendo esta uma necessidade por interação social inerente do ser humano, que estimula a produção linguística inicial. Ela manifesta sua intenção comunicativa inicialmente com gesto e atenção visual e, com o início da fala, as habilidades pragmáticas se

(1) Fonoaudióloga; Mestre e Doutoranda em Educação Especial pela Universidade Federal de São Carlos - UFSCar.

(2) Psicóloga; Professora Permanente do Programa de Pós-Graduação em Educação Especial da Universidade Federal de São Carlos - UFSCar, São Carlos, SP.

(3) Psicóloga; Professora Livre-docente do Departamento de Fonoaudiologia da Faculdade de Odontologia de Bauru, Universidade de São Paulo - FOB - USP, Departamento de Fonoaudiologia, Bauru - SP.

Conflito de interesses: inexistente manifestam de maneira mais produtiva, por meio de nomeações, comentários, pedidos de informação, de objetos e de atenção, respostas, protestos e saudações ${ }^{1-3}$.

Depois dos dois anos, aproximadamente 40\% das crianças mantêm o tópico do discurso na conversação e aos três anos esse número chega em $50 \%$. Entre três anos e meio e quatro anos, as crianças já demonstram habilidades na manutenção do tópico, realizando um maior número de comentários no discurso ${ }^{4}$. A participação nas trocas verbais requer habilidades conversacionais básicas, como a capacidade para iniciar e interagir e para responder apropriadamente e manter a interação. As habilidades de interação verbal são o centro das teorias de desenvolvimento de linguagem no contexto social. Durante a interação social, as crianças aprendem como usar a linguagem e também suas necessidades sociais ${ }^{5}$. 
No entanto, o desenvolvimento normal nem sempre ocorre e, não é raro, o surgimento de alterações no desenvolvimento de linguagem, como os Distúrbios Específicos de Linguagem (DEL).

Alguns estudos têm observado que as crianças com DEL possuem habilidades discursivas limitadas, o que pode acarretar consequências sociais ruins no processo de interação social de forma geral, interferindo também na qualidade de vida ${ }^{4,6-8}$. Demonstram algumas funções conversacionais essenciais, porém, muitas vezes, se expressam de maneira menos efetiva. Estas crianças com DEL possuem maior probabilidade para desenvolver problemas pragmáticos ${ }^{4}$.

A natureza das dificuldades pragmáticas e sua extensão parecem diferir entre as patologias da comunicação. O uso funcional da linguagem é afetado por habilidades sociais e seu déficit pode ser percebido na intenção comunicativa, nas pressuposições e no gerenciamento do discurso ${ }^{9}$.

Habilidades sociais são consideradas fatores aprendidos para a emissão de comportamentos adequados, diante das diversas situações do cotidiano. O termo habilidade social refere-se ao desempenho do indivíduo em interações sociais ${ }^{10}$. A aprendizagem das habilidades sociais se inicia na infância, primeiramente com a família e depois em outros contextos (escolar comunitário); o familiar constitui a base da estimulação inicial dos padrões de relacionamento e competência social ${ }^{10-14}$. O ambiente familiar e escolar constituem contextos privilegiados para o desenvolvimento das capacidades comunicativas e linguísticas da criança, necessárias a um futuro desempenho social e acadêmico com sucesso ${ }^{10-12}$.

Alguns estudos especializados ${ }^{15-17}$, assim como dados da prática clínica, apontam para um impacto das alterações da linguagem nos processos de desenvolvimento psicológico e social do indivíduo.

As respostas das crianças com DEL são um foco importante no estudo da pragmática. Comparando pares de crianças de mesma idade com DEL e em desenvolvimento normal de linguagem, os primeiros respondem inapropriadamente em maior quantidade. Quando comparados utilizando-se a extensão média dos enunciados (MLU), as crianças com DEL fracassam nas elaborações mais complexas e nas habilidades de estruturar suas respostas. Dessa forma, as crianças com DEL respondem, porém de forma linguisticamente atípica. Além do uso de estruturas linguísticas menos sofisticadas que seus pares nesta população $0^{1,9,17,18}$.

Uma criança com habilidades comunicativas reduzidas demonstra dificuldade em relacionar-se com os irmãos e com seus pares, pois a maneira pela qual utiliza a linguagem foge ao padrão esperado. Dessa forma, a criança pode tornar-se tímida, ausente, isolada, além de sofrer com os insultos e gozações de seus colegas. Outro fator, não menos importante, é com relação à escolarização da criança com dificuldades de comunicação, o professor deve estar atento, pois as habilidades que envolvem a linguagem, tal como a leitura, pode ser bastante prejudicada. E em casos mais graves, a criança pode precisar de escolarização especial ${ }^{18}$. Dessa maneira, o objetivo do presente estudo foi avaliar as habilidades pragmáticas e sociais em crianças com Distúrbio Específico de Linguagem (DEL).

\section{MÉTODO}

Participaram desse estudo 18 escolares, de ambos os gêneros, com idade entre 84 meses (7:0 anos) e 108 meses (9:11 anos).

Metade deles $(n=10)$, que constituíram o Grupo Experimental (GE), foram selecionados dentre aqueles que receberam o diagnóstico de Distúrbio Específico de Linguagem (DEL), por meio da Clínica de Diagnóstico dos Distúrbios da Comunicação Humana.

Considerando o diagnóstico de DEL, os participantes apresentaram os seguintes fatores de inclusão: desempenho linguístico abaixo do esperado para a idade mental e cronológica, considerando-se a expressão e/ou compreensão da linguagem oral; limiares auditivos dentro dos padrões de normalidade; ausência de problemas comportamentais e/ou emocionais; desempenho cognitivo de acordo com a normalidade, ou ainda, discrepância entre o desempenho das habilidades cognitivas verbais e não-verbais.

Como critério de inclusão neste grupo, além dos anteriormente citados, inerentes ao diagnóstico de DEL, também se exigiu que a criança nunca tivesse sido submetida à intervenção fonoaudiológica e possuísse idade igual ou superior a sete anos, de modo a garantir a persistência das alterações de linguagem.

O Grupo Controle (GC) foi constituído por nove escolares, com desenvolvimento cognitivo, de linguagem e de audição normais para a idade cronológica, os quais foram pareados aos do GE atendendo ao critério idade cronológica. Essas crianças foram selecionadas em escolas públicas da cidade de São Carlos - SP e submetidas à triagem fonoaudiológica que envolveu entrevista com os pais e análise de linguagem por amostra (conversação). As crianças selecionadas não apresentavam queixas referentes ao desenvolvimento de linguagem, motor, social e escolar, assim como apresentavam desenvolvimento de linguagem 
Tabela 1 - Caracterização dos participantes quanto ao gênero, idade e subgrupos

\begin{tabular}{|c|c|c|c|c|c|c|}
\hline \multirow[t]{2}{*}{ Grupos } & \multicolumn{2}{|c|}{ Gênero (n=18) } & \multirow{2}{*}{$\begin{array}{c}\text { Idade } \\
\text { cronológica } \\
\text { (média) }\end{array}$} & \multicolumn{3}{|c|}{$\begin{array}{c}\text { Subgrupos } \\
\text { (por faixa etária) }\end{array}$} \\
\hline & M & $\mathbf{F}$ & & GI & GII & GIII \\
\hline GE & 6 & 3 & 8,0 & 5 & 3 & 1 \\
\hline GC & 6 & 3 & 7,8 & 5 & 3 & 1 \\
\hline Total & 12 & 6 & - & 10 & 4 & 2 \\
\hline
\end{tabular}

compatível com a normalidade ${ }^{19}$. A Tabela 1 caracteriza os participantes de acordo com o gênero, idade e subgrupos, que foram divididos em: Grupo I - (7a - 7a e 11m), Grupo II - (8a - 8a e $11 \mathrm{~m})$ e Grupo III - (9a - 9a e 11m).

A coleta de dados foi realizada em dois dias diferentes, sendo que cada criança (GC e GE) foi filmada por duas vezes, cada vez com 15 minutos de duração.

A orientação seguida para as filmagens foi de distribuir os brinquedos (jogos) na mesa e, ao sentar com a criança, convidá-la a brincar com os jogos ali expostos. Durante as filmagens foi solicitado que o interlocutor adulto (sexo feminino) seguisse um roteiro de interação de maneira a observar presença ou ausência dos componentes verbais de conteúdo (fazer e responder perguntas; iniciar, manter e encerrar conversação), verbais de forma (duração, volume, entonação) e não verbais (contato visual, gestos, sorriso, expressões faciais, movimentos de cabeça, postura corporal) das Habilidades Sociais de Comunicação (HSC).

Todas as crianças tiveram suas atividades gravadas em áudio e vídeo. As falas da criança e do adulto durante as filmagens foram transcritas e analisadas, bem como foram observados os gestos com propósitos comunicativos (sim e não com balanço de cabeça).

Dois juízes (fonoaudiólogos) treinados analisaram as filmagens das crianças frente à situação estruturada de interação, anotando no Protocolo de Registro da Avaliação do Desempenho (PRAD) elaborado pela pesquisadora, presença ou ausência dos comportamentos descritos acima de acordo com a frequência de resposta ( ${ }^{*}$ Satisfatório: $40<\mathrm{FqR}<$ <0; *Insatisfatório: $0<\mathrm{FqR}<39,9$ ).

As habilidades pragmáticas foram caracterizadas de acordo com as habilidades conversacionais (inicia turnos; responde/mantém; não responde/ mantém; turnos simples; turnos expansivos; turnos coerentes e turnos incoerentes) e função comunicativa interativa (uso de expressões sociais para iniciar ou encerrar a interação). Para a análise foram categorizadas em Respostas Adequadas (respondem adequadamente ao diálogo de acordo com as habilidades conversacionais e funções comunicativas) e Respostas Inadequadas (respondem inadequadamente ao diálogo de acordo com as habilidades conversacionais e funções comunicativas).

A fidedignidade interobservadores foi calculada segundo a fórmula abaixo 20

$$
\mathrm{IC}=\frac{\sum \mathrm{A}}{\Sigma \mathrm{A}+\overline{\Sigma D}} \times 100
$$

Legenda: IC (Índice de Concordância), $\Sigma$ A (somatória dos acordos), $\Sigma \mathrm{D}$ (somatória dos desacordos)

Foram considerados fidedignos os dados com, no mínimo, $75 \%$ de concordância.

O projeto desse estudo foi submetido ao Comitê de Ética em Pesquisa com Seres Humanos da Faculdade de Odontologia de Bauru da Universidade de São Paulo, recebendo aprovação de número $21 / 2011$ e todos os responsáveis pelos participantes assinaram um termo de consentimento livre e esclarecido concordando com as normas do estudo.

Para a análise estatística foi utilizado o Teste-T Student (Excel 2010), considerando $p<0,05$, com o objetivo de comparar os resultados obtidos tanto nos grupos quanto subgrupos.

\section{RESULTADOS}

Para a análise das filmagens da conversação criança - adulto, com objetivo de avaliação da pragmática, as respostas foram classificadas em categorias e agrupadas em Repostas Adequadas (RA) e Repostas Inadequadas (RI), sempre de acordo com o contexto comunicativo estabelecido. Observa-se, na Tabela 2, que o GC apresentou média significantemente maior que o GE para o uso de RA e o GE apresentou média significantemente maior que o GC para o uso de RI. O GC diminuiu o uso de RI com o aumento da idade, quanto que GE manteve o uso de RI. 
Exemplos de Respostas Inadequadas:

Adulto: O que é isso na sua mão?

Criança: Na casa vó minha...

Adulto: Qual dos brinquedos que estão aqui você mais gosta?

Criança: Brinquedo na loja...
Adulto: O que você gostaria de fazer hoje?

Criança: Amanhã fui brincar João.

Adulto: Tchau M... Te espero amanhã!

Criança: Ontem eu venho!

Tabela 2 - Média das respostas adequadas e inadequadas dos grupos e subgrupos

\begin{tabular}{c|c|c|c}
\hline \multirow{2}{*}{ Grupos } & Subgrupos & $\begin{array}{c}\text { Respostas Adequadas } \\
\text { (RA - média) }\end{array}$ & $\begin{array}{c}\text { Respostas Inadequadas } \\
\text { (RI - média) }\end{array}$ \\
\hline \multirow{4}{*}{ GE } & GI & 69,30 & 7,4 \\
\cline { 2 - 4 } & GII & 70,10 & 7,35 \\
\cline { 2 - 4 } & GIII & 71,20 & 7,35 \\
\cline { 2 - 4 } & Média do GE & $\mathbf{7 0 , 8 6}$ & $\mathbf{7 , 3 6}$ \\
\cline { 2 - 4 } & GI & 93 & 2,03 \\
\cline { 2 - 4 } & GII & 94 & 1,93 \\
\cline { 2 - 4 } & GIII & 94 & $\mathbf{1 , 3 2}$ \\
\hline \multirow{3}{*}{ Sig (p<0,05) } & Média do GC & $\mathbf{9 3 , 6 6}$ & $\mathbf{0 , 0 1 1 4 2 2}$ \\
\hline
\end{tabular}

$\mathrm{Na}$ tabela 3 são apresentados os resultados obtidos com relação a análise das filmagens em situação estruturada de interação. As frequências da respostas foram agrupadas em Satisfatórias $(40<\mathrm{FqR}<90)$ e Insatisfatórias $(0<\mathrm{FqR}<39,9)$, considerando os componentes das habilidades sociais de comunicação: verbais de conteúdo, verbais de forma e não verbais. Considerando a análise estatística, com $p<0,05$, pode-se observar que o GC apresentou melhor desempenho na situação de interação que o GE, mostrando diferença significante em todos os componentes das HSC.

Tabela 3 - Frequência de respostas de acordo com os componentes das habilidades sociais de comunicação

\begin{tabular}{c|c|c|c|c|c|c|c}
\hline Grupos & \multicolumn{7}{c}{ Componentes } \\
\cline { 3 - 8 } & \multirow{3}{*}{ Subgrupos } & Verbais de Conteúdo & \multicolumn{2}{c}{ Verbais de Forma } & \multicolumn{2}{c}{ Não Verbais } \\
\cline { 3 - 8 } & & $\mathbf{S}$ & $\mathbf{I}$ & $\mathbf{S}$ & $\mathbf{I}$ & $\mathbf{S}$ & $\mathbf{I}$ \\
\hline \multirow{4}{*}{ GE } & GI & 40,1 & 34,2 & 40,5 & 30,5 & 47,5 & 39,7 \\
\cline { 2 - 8 } & GII & 40,6 & 32,8 & 42,8 & 28,6 & 47,0 & 38,7 \\
\cline { 2 - 8 } & GIII & 43,6 & 35,6 & 48,5 & 25,4 & 46,2 & 36,2 \\
\cline { 2 - 8 } & Média do GE & $\mathbf{4 0 , 7}$ & $\mathbf{3 4 , 2}$ & $\mathbf{4 3 , 9}$ & $\mathbf{2 8 , 1}$ & $\mathbf{4 6 , 9}$ & $\mathbf{3 8 , 2}$ \\
\hline \multirow{3}{*}{ GC } & GI & 48,3 & 28,0 & 56 & 24,5 & 58 & 23,3 \\
\cline { 2 - 8 } & GII & 51,3 & 26,5 & 58,3 & 22,2 & 59,3 & 21,6 \\
\cline { 2 - 8 } & GIII & 53,7 & 24,8 & 61,7 & 20,8 & 62 & 17,9 \\
\cline { 2 - 8 } & Média do GC & $\mathbf{5 1 , 1}$ & $\mathbf{2 6 , 4}$ & $\mathbf{5 8 , 6}$ & $\mathbf{2 2 , 5}$ & $\mathbf{5 9 , 7}$ & $\mathbf{2 0 , 9}$ \\
\hline $\mathbf{( p < 0 , 0 5 )}$ & & $\mathbf{0 , 0 0 0 3 2 2}$ & $\mathbf{0 , 0 3 6 0 9 5}$ & $\mathbf{0 , 0 0 2 6 9 7}$ & $\mathbf{0 , 0 0 9 1 4 6}$ & $\mathbf{0 , 0 1 4 3 1 1}$ & $\mathbf{0 , 0 0 1 0 3 1}$ \\
\hline
\end{tabular}

*Satisfatório: $40<$ FqR < 90; *Insatisfatório: $0<$ FqR < 39,9 


\section{DISCUSSÃO}

Com o início das habilidades de respostas, as crianças menores comumente apresentam respostas semântica e pragmaticamente inapropriadas. Estas crianças usam a entonação para realizarem suas respostas, pois esta serve como pista para as respostas de interrogativos. Com o aumento do léxico há uma melhora na qualidade das respostas, o que garante que, a partir dos três anos, as crianças sejam capazes de apresentar várias formas de respostas ${ }^{1,4}$. Dessa forma, as respostas das crianças com DEL são importantes para estudos da pragmática.

Ao comparar os Grupos GE e GC (tabela 2), pode-se observar que GE apresentou maior quantidade de respostas inadequadas que o GC. Quando comparados utilizando-se a extensão média dos enunciados, as crianças com DEL fracassaram nas elaborações mais complexas e nas habilidades de estruturar suas respostas. Ou seja, as crianças com DEL responderam, porém de forma linguisticamente atípica. Ainda nesse estudo, foi observado que o aumento da idade salientou as diferenças entre GE e GC. O GE foi menos efetivo na comunicação, ao passo que o GC apresentou habilidades conversacionais mais elaboradas.

O desenvolvimento das capacidades comunicativas e linguísticas da criança é necessário para um futuro desempenho social e acadêmico com sucesso. Como as crianças com DEL possuem habilidades discursivas limitadas, o processo de interação social pode estar prejudicado, acarretando déficits em habilidades sociais, ou seja, as alterações observadas nesta população interferem na vida social dessas crianças, como uma barreira comunicativa.

Os dados obtidos corroboram com resultados publicados em pesquisas da área ${ }^{1-5,8,9,9,17-19}$, que mostraram que as crianças com DEL, em comparação com crianças em fase normal de desenvolvimento da linguagem, demonstraram algumas funções conversacionais essenciais, porém, muitas vezes, se expressaram de maneira menos efetiva. Os dados indicaram ainda que as crianças do GC passaram a ficar mais ativas na comunicação nas idades maiores, enquanto que GP não demonstrou a mesma evolução referida para o GC. Foi observado que as crianças com DEL se comparadas aos seus pares em desenvolvimento normal não apresentaram alterações pragmáticas evidentes, porém, demonstraram uso de estruturas linguísticas menos sofisticadas.

O presente estudo pode relacionar a frequência de respostas com os componentes verbais de conteúdo, verbais de forma e não verbais das habilidades sociais (tabela 3). Mostrando que, o GC apresentou maior quantidade de respostas satisfatórias que o GE, estando dentro dos padrões esperados para um bom desempenho social, enquanto que o GE apresentou menor iniciativa e desenvoltura na situação estruturada de interação. De acordo com a análise estatística, pode-se observar que houve diferença significante entre os grupos. Deve-se estar atendo às crianças com habilidades conversacionais reduzidas, pois estas podem apresentar dificuldades em relacionar-se, além de sofrer com insultos e gozações por seus pares.

\section{CONCLUSÃO}

Dentro das limitações desse estudo, pode-se observar a relação entre as habilidades pragmáticas e os déficits em habilidades sociais em crianças com Distúrbio Específico de Linguagem. Ressalta-se a importância de novas pesquisas que possam elaborar métodos de avaliação e intervenção que busquem tanto a melhoria nos aspectos de fala e linguagem quanto nas relações interpessoais. 


\begin{abstract}
Purpose: to evaluate the social skills and pragmatic communication in children with Specific Language Impairment. Method: this study involved 18 children between seven and nine year old, with half $(n=9)$ being the Experimental Group (EG) and the other half $(n=9)$ the Control Group CG. Both groups were comprised of three girls and six boys. Children were evaluated as for pragmatic abilities through a spontaneous speech footage. Then, recorded in structured situations of interaction with an unfamiliar adult female. Responses were classified in categories and grouped in Adequate Response (AR) and Inadequate Responses (IR), always according to the communicative context established. Results: it was observed that the greater is the limitation in pragmatic abilities, initiative and performance are worst in interpersonal relationships. There was a significant difference between EG and CG, indicating that the EG had underperformed the CG in both pragmatic and social skills. Conclusion: we stress out the importance of developing methods regarding intervention that seek to improve the aspects of speech, language, and interpersonal relationships.
\end{abstract}

KEYWORDS: Communication; Language Development; Child Language; Interpersonal Relations

\section{REFERÊNCIAS}

1. Béfi-Lopes DM. Diagnóstico e princípios terapêuticos em distúrbio específico de linguagem - do fenótipo para o genótipo [resumo 24]. Pró-Fono Revista de Atualização Científica [Internet]. 2008 [citado 2008 out 13];20(Supl):72-5. [Apresentado no II Congresso Brasileiro de Fonoaudiologia e Genética dos Distúrbios da Comunicação; 2008; Fortaleza, Ceará]. Disponível em: www. revistaprofono.com.br.

2. Brinton B, Fujiki M. A comparison of requestresponse sequences in the discourse of normal and language-disordered children. J. Speech Hear. Dis. 1982;47:57-62, feb.

3. Roth FP, Spekman NJ. Assessing the pragmatic abilities of children, part 1: organizational framework and assessment parameters. J. Speech Hear. Dis. 1984;49:2-11.

4. Rocha LC, Befi-Lopes DM. Análise pragmática das respostas de crianças com e sem distúrbio específico de linguagem. Pró-Fono Rev. Atual. Cient. 2006;18:229-39.

5. Hadley PA, Rice ML. Conversational responsiveness of speech and language impaired preschoolers. J. Speech Hear. Res. 1991;34:1304-17.

6. Conti-Ramsden G, Botting N. Social difficulties and victimization in children with SLI at 11 years of age. J. Speech Lang. Hear. Res. 2004;47(1):145-61.

7. Hart $\mathrm{KI}$, Fujiki $\mathrm{M}$, Brinton $\mathrm{B}$, Hart $\mathrm{CH}$. The relationship between social behavior and severity of language impairment. J. Speech Lang. Hear. Res. 2004;47(3):647-62.
8. Béfi-Lopes DM, Bento ACP, Perissinoto J. Narração de histórias por crianças com distúrbio específico de linguagem. Pró-Fono Revista de Atualização Científica. 2008;20(2):93-8.

9. Béfi-Lopes DM, Pereira ACS, Bento ACP. Representaçãofonológica em criançascom Distúrbio Específico de Linguagem (DEL). Pró-Fono Revista de Atualização Científica. 2010;22(3):305-10.

10. Del Prette A, Del Prette ZAP, Barreto MCM. Habilidades sociales en la formación del psicólogo: Análisis de un programa de intervención. Psicología Conductual (Espanha). 1999, 7: 27-47.

11. Del Prette ZAP, Del Prette A. Habilidades sociais: Uma área em desenvolvimento. Psicologia Reflexão e Crítica. 1997;9: 233-55.

12. Del Prette ZAP, Del Prette A. Habilidades sociais e dificuldades de aprendizagem: Teoria e pesquisa sob um enfoque multimodal. Em Del Prette A. Del Prette ZAP. (Orgs). Habilidades sociais, desenvolvimento e aprendizagem: Questões conceituais, avaliação e intervenção. Campinas, SP: Alínea. 2003, pp. 167-206.

13. Bolsoni-Silva AT, Marturano EM. Práticas educativas e problemas de comportamento: Uma análise à luz das habilidades sociais. Estudos de Psicologia. 2002; 7(2): 227-35.

14. Gomide PIC. Estilos parentais e comportamento anti-social. Em Del Prette A, Del Prette ZAP (Orgs.), Habilidades sociais, desenvolvimento e aprendizagem. Campinas: Alínea. 2003, pp. 21-60.

15. Rutter M, Mawhood L. Reliability and validity of a psychosocial axis in child with language impairment. J Child Psychol Psychiatry. 1991;41:561-78. 
16. Wood J, Cowan P, Baker RB. Behavior problems and peer rejection in preschool boys and girls. Journal of Genetic Psychology. 2002;163:72-88.

17. Carlino FC. Relação entre Inteligibilidade de Fala e Habilidades Sociais de Comunicação em Crianças com Desvio Fonológico. Dissertação de mestrado. Programa de Pós-Graduação em Educação Especial, Universidade Federal de São Carlos-UFSCar. São Carlos, Brasil. 2010, 134p.
18. Syder D. An Introduction to Communication Disorders. Livraria e Editora Revinter Ltda. 1997, pp. 1-72.

19. Zorzi JL. A intervenção Fonoaudiológica nas Alterações da Linguagem Infantil. (2 ${ }^{a}$ edição). Rio de Janeiro. Editora Revinter Ltda. 2008, pp. 75-81.

20. Hersen M, Barlow DH. Single case experimental designs: strategies for studying behavior change. $2^{\circ}$ edição, New York: Pergamon Press, 1982.

Endereço para correspondência:

Fabiana Cristina Carlino

Rua Marcolino Lopes Barreto, 2886

Vila Costa do Sol - São Carlos - SP

CEP: 13566-210

E-mail: fccarlino.usp@gmail.com 